\title{
Consensus recommendations on the classification, definition and diagnostic criteria of hip-related pain in young and middle-aged active adults from the International Hip-related Pain Research Network, Zurich 2018
}

\author{
Michael P Reiman 다, ${ }^{1}$ Rintje Agricola, ${ }^{2}$ Joanne L Kemp 지, ${ }^{3}$ Joshua J Heerey (다, ${ }^{3}$ \\ Adam Weir, ${ }^{4,5}$ Pim van Klij (1) , ${ }^{2}$ Ara Kassarjian, ${ }^{6,7}$ Andrea Britt Mosler (ㄷ), ${ }^{3}$ \\ Eva Ageberg (1) ${ }^{8}$ Per Hölmich, ${ }^{9}$ Kristian Marstrand Warholm (10, , ${ }^{10}$ \\ Damian Griffin, ${ }^{11,12}$ Sue Mayes, ${ }^{3}$ Karim M Khan 두, ${ }^{13}$ Kay M Crossley (1), ${ }^{3}$ \\ Mario Bizzini, ${ }^{14}$ Nancy Bloom, ${ }^{15}$ Nicola C Casartelli 이, ${ }^{16,17}$ Laura E Diamond (1) ${ }^{18}$ \\ Stephanie Di Stasi $10{ }^{19}{ }^{19}$ Michael Drew ${ }_{1}^{20,21}$ Daniel J Friedman, ${ }^{22}$ Matthew Freke, ${ }_{1}{ }^{23}$ \\ Boris Gojanovic (ㄷ) ${ }^{24,25}$ Sion Glyn-Jones, ${ }^{26}$ Marcie Harris-Hayes (ㄷ) , ${ }^{15}$ \\ Michael A Hunt, ${ }^{27}$ Franco M Impellizzeri (i), ${ }^{28}$ Lasse Ishøi @ ${ }^{9}$ Denise M Jones, ${ }^{3}$ \\ Matthew G King (1) , ${ }^{3}$ Peter R Lawrenson 이, ${ }^{29}$ Michael Leunig, ${ }^{30}$ Cara L Lewis (ㄷ, ${ }^{31}$ \\ Nicolas Mathieu, ${ }^{32}$ Håvard Moksnes, ${ }^{33}$ May-Arna Risberg, ${ }^{34,35}$ \\ Mark James Scholes (1), ${ }^{3}$ Adam I Semciw, ${ }^{3}$ Andreas Serner $(1),{ }^{36}$ \\ Kristian Thorborg (1) , ${ }^{9}$ Tobias Wörner (1), ${ }^{37}$ Hendrik Paulus Dijkstra ${ }^{36,38}$
}

\begin{abstract}
- Additional material is published online only. To view please visit the journal online (http://dx.doi.org/10.1136/ bjsports-2019-101453).
\end{abstract}

For numbered affiliations see end of article.

Correspondence to Dr Michael P Reiman, Orthopedic Surgery, Duke University Medical Center, Durham, NC 27710, USA; reiman.michael@gmail.com

Accepted 1 January 2020 Published Online First 20 January 2020
Check for updates

(C) Author(s) (or their employer(s)) 2020. No commercial re-use. See rights and permissions. Published by BMJ.

To cite: Reiman MP Agricola R, Kemp JL, et al. Br J Sports Med 2020;54:631-641

\section{ABSTRACT}

There is no agreement on how to classify, define or diagnose hip-related pain-a common cause of hip and groin pain in young and middle-aged active adults. This complicates the work of clinicians and researchers. The International Hip-related Pain Research Network consensus group met in November 2018 in Zurich aiming to make recommendations on how to classify, define and diagnose hip disease in young and middle-aged active adults with hip-related pain as the main symptom. Prior to the meeting we performed a scoping review of electronic databases in June 2018 to determine the definition, epidemiology and diagnosis of hip conditions in young and middle-aged active adults presenting with hip-related pain. We developed and presented evidencebased statements for these to a panel of 37 experts for discussion and consensus agreement. Both nonmusculoskeletal and serious hip pathological conditions (eg, tumours, infections, stress fractures, slipped capital femoral epiphysis), as well as competing musculoskeletal conditions (eg, lumbar spine) should be excluded when diagnosing hip-related pain in young and middle-aged active adults. The most common hip conditions in young and middle-aged active adults presenting with hiprelated pain are: (1) femoroacetabular impingement (FAI) syndrome, (2) acetabular dysplasia and/or hip instability and (3) other conditions without a distinct osseous morphology (labral, chondral and/or ligamentum teres conditions), and that these terms are used in research and clinical practice. Clinical examination and diagnostic imaging have limited diagnostic utility; a comprehensive approach is therefore essential. A negative flexion-adduction-internal rotation test helps rule out hip-related pain although its clinical utility is limited. Anteroposterior pelvis and lateral femoral head-neck radiographs are the initial diagnostic imaging of choice-advanced imaging should be performed only when requiring additional detail of bony or softtissue morphology (eg, for definitive diagnosis, research setting or when planning surgery). We recommend clear, detailed and consistent methodology of bony morphology outcome measures (definition, measurement and statistical reporting) in research. Future research on conditions with hip-related pain as the main symptom should include high-quality prospective studies on aetiology and prognosis. The most common hip conditions in active adults presenting with hip-related pain are: (1) FAl syndrome, (2) acetabular dysplasia and/ or hip instability and (3) other conditions without distinct osseous morphology including labral, chondral and/or ligamentum teres conditions. The last category should not be confused with the incidental imaging findings of labral, chondral and/or ligamentum teres pathology in asymptomatic people. Future research should refine our current recommendations by determining the clinical utility of clinical examination and diagnostic imaging in prospective studies.

\section{INTRODUCTION}

Young and middle-aged active adults with hip and groin pain often present with a confusing overlap of signs and symptoms. ${ }^{1}$ There is no consensus on how to define or classify hip disease with different and overlapping intra-articular and extra-articular contributors to symptoms, in an anatomically complex region. Several researchers and consensus 
groups have previously attempted to define aspects of hip and groin pain..$^{2-7}$ Among these are consensus agreements on the terminology and definitions for groin pain in athletes ${ }^{2}$ and on femoroacetabular impingement (FAI) syndrome. ${ }^{3}$

The Doha Agreement Meeting on terminology and definitions in groin pain in athletes divided groin pain into three major categories: clinical entities for groin pain (adductor-related, iliopsoas-related, inguinal-related and pubic-related), hip-related and other causes of groin pain in athletes. ${ }^{2}$ The Doha Agreement concluded that "hip-related pain can be difficult to distinguish from other causes and that it may coexist with other types of groin pain." A detailed classification of possible causes of hiprelated pain in athletes was considered to be outside the scope of the Doha Agreement process. ${ }^{2}$ This difficulty was related to the inability for hip examinations to assist in differential diagnosis, and probably only being useful for screening ${ }^{3}$-as most clinical tests have good sensitivity but poor specificity. ${ }^{2}$

There is no consensus on how to classify hip-related pain in young and middle-aged active adults, with prior studies focusing on classifying individual conditions, such as FAI syndrome, ${ }^{3} 4$ 6-9 acetabular dysplasia, ${ }^{10}$ labral tear, ${ }^{8}$ chondral lesions and ligamentum teres tears. ${ }^{11}$ Each of these conditions is described in detail in online supplementary appendix 1. Briefly, in FAI syndrome, the repetitive mechanical loading of cartilage between the femoral head and acetabular rim is suggested to contribute to acetabular labral and chondrolabral findings, as well as early osteoarthritis. ${ }^{12} 13$ As such, FAI syndrome was defined as a motion-related clinical disorder of the hip with a triad of symptoms, clinical signs and imaging findings (typically cam, pincer or mixed morphology) that represents symptomatic premature contact between the proximal femur and the acetabulum. ${ }^{3}$ The primary symptom of FAI syndrome has traditionally been described as groin pain, ${ }^{3} 1415$ although pain may also be felt in the back, buttock or thigh. ${ }^{14}{ }^{16}$ Not having groin pain has been suggested to help exclude FAI syndrome and/or labral tear findings in patients with hip pain. ${ }^{17-19}$ Acetabular dysplasia refers to misalignment between the femoral head and the acetabulum secondary to changes in their shape, size and orientation, ${ }^{10}$ which may result in instability and overload of the acetabular rim during normal activities. ${ }^{2021}$

Differential diagnosis of any condition requires exclusion of more serious musculoskeletal and non-musculoskeletal pathology, as well screening appropriate body regions. For hiprelated pain in young active adults, this could for example include Perthes' disease, slipped capital femoral epiphysis (SCFE), infection, osteoarthritis, tumours and fractures. While it is beyond the scope and purpose of detailing the process of helping to exclude these pathological concerns, the reader is referred to other recent works detailing this process. ${ }^{22} 23$

A proper classification of non-arthritic hip disease in active adults into well-defined hip conditions will provide more clarity for clinicians and researchers. This is critically important due to the significant increase in the diagnosis of hip conditions and incidental imaging findings in athletes, ${ }^{24} 25$ as well as arthroscopic hip surgery in general. ${ }^{26-28}$ There is a lack of current best evidence on the classification of hip-related pain, partly due to the heterogeneity in the definition of hip-related pain. Consensus, informed by current best evidence, is needed to provide clarity for clinicians interpreting the findings of research papers, particularly with the increasing knowledge of incidental imaging findings in young and middle-aged active adults. ${ }^{24} 25$

The International Hip-related Pain Research Network (IHiPRN) was established in 2017 to facilitate collaboration across research groups and disciplines and to improve knowledge dissemination of hip-related pain to clinicians. The specific focus of the IHiPRN is 'non-red flag pain originating from the hip joint in young and middle-aged active adults'. We identified four topics on hip-related pain for consensus agreement: classification of hip pain, patient-reported outcomes, standardised measurement of physical capacity and physiotherapist-led treatment.

In this paper, we first describe the general consensus process applied to all topics in this series. We then make recommendations on the first topic on how to classify, define and diagnose hip disease in young and middle-aged active adults, with hiprelated pain as the main symptom. Other papers in this series will use this classification of hip-related pain in the assessment of (1) patient-reported outcome measures, (2) standardised measurement of physical capacity and (3) physiotherapist-led treatment.

\section{METHODS}

\section{Literature review}

For the first key area on the classification, definition and diagnostic criteria of hip-related pain in young and middle-aged active adults, working group 1 performed a literature review to identify gaps in the literature to formulate consensus recommendations and provide research recommendations for future study. A summary of the literature review is contained in online supplementary appendix 1. Using components of a scoping review framework, ${ }^{29}$ our inclusion and exclusion criteria were the following:

- Study design: Systematic reviews, intervention and observational studies (prospective or retrospective) with a study population of at least 10 young and middle-aged active adults and published in English language, peer-reviewed journals. We excluded conference abstracts, case studies, narrative reviews and non-peer-reviewed studies.

- Population: Research participants diagnosed with hiprelated pain or related condition. Participants with primary osteoarthritis, SCFE, Legg-Calve-Perthes or extra-articular condition were excluded as these are not the conditions identified as being of primary prevalence in participants of this age range and pain description.

- Outcome: Definition, epidemiology, clinical and/or imaging diagnosis of any hip-related pain condition.

\section{Consensus meeting}

The consensus process used is shown in figure 1 .

\section{Selection of expert group members}

The IHiPRN leadership group (JLK, KMC, MB, ABM, CLL and KMK) met in January 2017 to identify potential expert group members. Experts were selected based on their track record of publications, and being current active researchers in the field of hip-related pain in young and middle-aged active adults. Many researchers were also clinicians in the field. Potential expert group members were invited to the first IHiPRN Consensus Meeting in Zurich in November 2018 (online supplementary appendix 2), and asked to identify additional experts for invitation.

Following this expression of interest, four key areas were identified as priorities for consensus. These four key areas were the following:

1. Classification of hip pain (including use of clinical tests and imaging).

2. Patient-reported outcome measures for hip pain (including hip-related measures and maybe others including pain/coping/fear/utility measures). 
Need for international consensus established January 2017

IHiPRN leadership group (JLK, KMC, MB, ABM, CLL, KMK) selected Expert Group members

Four key priority areas identified and four working groups established

- Leadership group identified experts to lead each of four working groups

- Working group members determined by discussion between leadership group and working group leaders

\section{Pre-agreement meeting process}

- $\quad$ Four working group prepared evidence summary and developed draft consensus recommendations

- Evidence summary and draft consensus recommendations presented to entire expert group prior to Zurich meeting

Agreement meeting process (November 2018)

- Working groups revised evidence summaries and draft consensus recommendations

- Working group leaders presented evidence summaries to all expert group members for discussion/further revision.

- 38 expert group members rated each recommendation on 10-point Likert scale

- Scores pooled and median for each recommendation determined

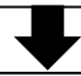

\section{Recommendations from four working groups form international consensus}

Figure 1 Flow chart of the consensus process. IHiPRN, International Hip-related Pain Research Network.

3. Standardised measurement of physical capacity in hip-related pain (including clinical measures, biomechanics, electromyography, physical activity, functional performance and return to sport).

4. Physiotherapist-led treatment of hip-related pain.

The leadership group then identified experts to lead each of the four working groups. These were MPR and RA (group 1), ABM and CLL (group 2), FI and JLK (group 3), JLK and MB (group 4). Members of the working groups were then determined following discussion between the leadership group and the working group leaders. The working group members for this paper were MPR, RA, JLK, HPD, JJH, AW, PvK, AK, ABM, EA, $\mathrm{PH}$ and KMW.

\section{Expert group demographics}

Areas of expertise among the expert group participants included physiotherapy, orthopaedic surgery, sport and exercise medicine, biomechanics, diagnostics, imaging and radiology, patient-reported outcome measures and exercise science. In addition, many participants were also expert clinicians who regularly treat young and middle-aged active adults with hiprelated pain.

\section{Premeeting process}

Prior to the meeting, the four working groups prepared evidence summaries (based on literature searches and syntheses) to help inform recommendations and underpin discussions at the consensus meeting. These evidence summaries and draft consensus recommendations were presented to all meeting participants via email at least 1 week prior to the Zurich meeting.

Consensus meeting process

At the beginning of the consensus meeting in Zurich, the working groups discussed and revised the draft consensus recommendations. Evidence summaries and consensus recommendations 
were then presented to, and discussed by the whole expert group, and further revisions were made. Following discussion, each participant was asked to vote on the recommendation on a 10-point Likert scale, where 0 was considered to be 'inappropriate' and 9 'appropriate', as described previously. ${ }^{31} 32$ Participants were blinded to each other's scoring. Scores were pooled and the median (IQR) for each recommendation was determined. Recommendations that scored $0-3$ were considered inappropriate, scored 4-6 were considered uncertain and scored 7-9 were considered appropriate. ${ }^{3132}$ The level of available evidence and the pooled voting score for the recommendation were considered to determine the final consensus recommendation.

\section{Reporting checklist}

Although there are common elements between the Appraisal of Guidelines, Research and Evaluation (AGREE) reporting checklist $^{33}$ and the aims of the Zurich meeting, the authors feel that AGREE is not the ideal way to share the work of the group on this topic. This paper discusses the consensus on the classification of hip-related pain and is one of the four above-mentioned key areas. The four key areas required varying methodological approaches due to the different nature of the content. Therefore, it was not appropriate to use a single checklist for each of these areas.

\section{Determining diagnostic utility for various clinical and radiological measures}

Diagnostic values (eg, sensitivity, specificity, likelihood ratios) for the various clinical and radiological measures are reported in the appendix. Since determination of clinical utility of these values is often poorly done or misunderstood, we have attempted to provide the reader with a graphical representation of how to potentially use and incorporate these values in clinical practice (eg, what clinical value should they be given). To do this requires examination of not only diagnostic accuracy but also magnitude of diagnostic probability shifts, ${ }^{34}$ precision and the quality of the study examining such measures.

Values used in this paper were stratified accordingly:

Magnitude: size of the shift in probability as previously defined. ${ }^{34}$

Precision: how repeatable are the results. This is defined as highest number divided by lowest number for the CI of the post-test probability value for each measure (eg, 95\% CI for a post-test probability value of 49 to 93 has a precision value of $93 / 49=1.9$ ). A lower value indicates higher precision. This was described previously as the upper-to-lower confidence limit ratio. ${ }^{35}$ A probability shift with a wide CI is imprecise and unstable. $^{35}$

Study Quality: high or low study quality based on previous systematic reviews that have quantified study quality. ${ }^{36} 37$ Charted as:

- High-quality study: $\Uparrow$

- Low-quality study: $\Downarrow$

Diagnostic utility was charted, and summaries of this for each condition are contained in figures $2-9$ in a $2 \times 2$ quadrant (magnitude $\times$ precision) accordingly:

- Red quadrant: values for both magnitude and precision are low, not typically recommended for clinical decisionmaking. These measures are Not Recommended.

- Yellow quadrant: values for either magnitude or precision are low. Caution is recommended for clinical decisionmaking. These measures are Cautiously Recommended.

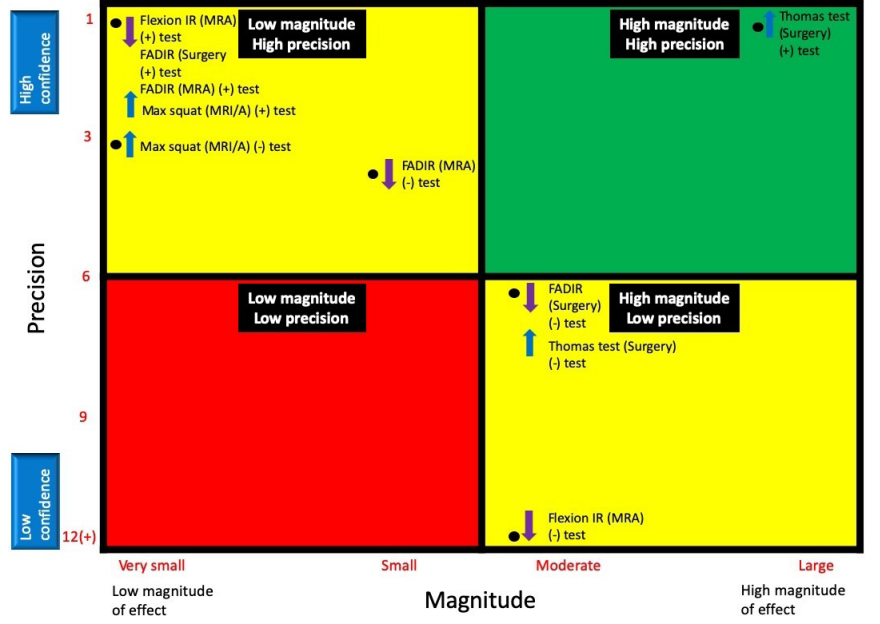

Figure 2 Magnitude and precision of clinical tests for diagnosis and screening of FAI syndrome and labral pathology. (+), positive test; (-), negative test; FADIR, flexion adduction internal rotation; FAI, femoroacetabular impingement; IR, internal rotation; MRA, magnetic resonance arthrogram. Reference standards are listed in ().

- Green quadrant: high magnitude and high precision. These measures are Recommended if study quality is high. Cautious recommendation for low-quality studies.

*We have drawn these figures in quadrants to help the reader, but just as with the quadrants in a cost-effectiveness curve, we appreciate the axis are continuous and there are no discrete cutoffs from 'low to high'.

\section{RESULTS}

Results of the consensus meeting: consensus meeting participants

The consensus meeting in Zurich, Switzerland (17-18 November 2018) was attended in person by 37 out of the 44 IHiPRN participants; one additional participant attended via videoconferencing.

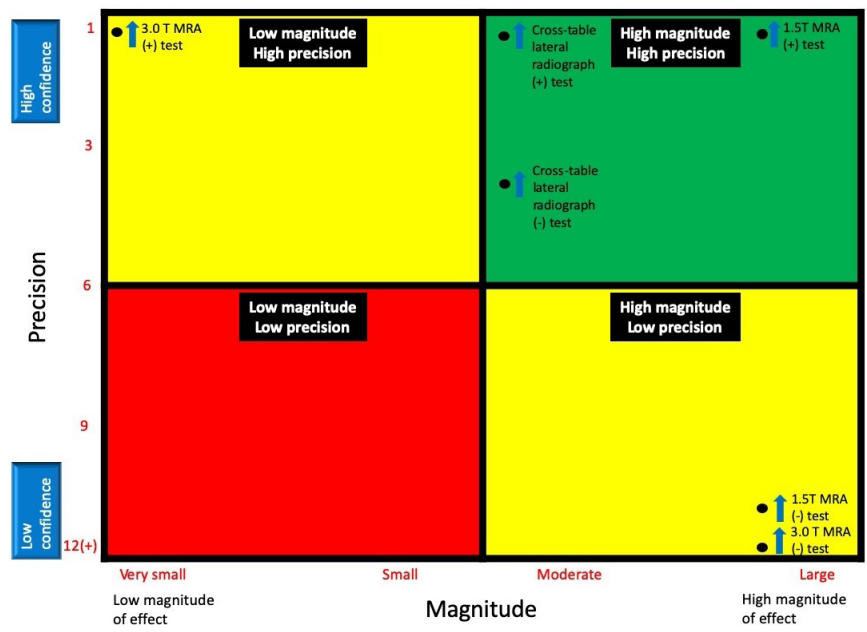

Figure 3 Magnitude and precision of imaging tests for diagnosis and screening of FAI syndrome. (+), positive test; (-), negative test; FAI, femoroacetabularimpingement; MRA, magnetic resonance arthrogram; T, Tesla. 


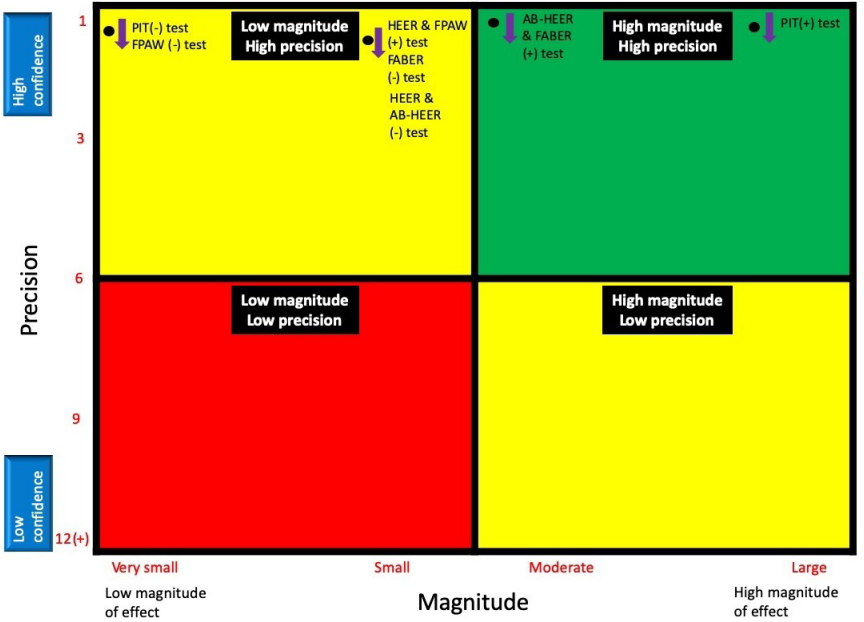

Figure 4 Magnitude and precision of clinical tests for diagnosis and screening of acetabular dysplasia and hip instability. (+),positive test; (-), negative test; AB-HEER, abduction-hyperextension-external rotation; FABER, flexion abduction external rotation; FPAW, foot progression angle walking; HEER, hyperextension-external rotation; PIT, prone instability test; (+) positive test, (-) negative test.

\section{Expert group demographics}

The expert group of 27 physiotherapists, 7 orthopaedic surgeons, 4 sport and exercise medicine physicians, 4 exercise scientists, 1 radiologist and 1 general medical doctor included 12 women and 32 men. Participants had a mean (SD) age of 41 (11) years and 20 (10) years of experience. Twenty-one participants were from Europe, 13 from Australia/New Zealand, 8 from North America and 2 from the Middle East. All participants were fluent English speakers.

\section{Literature search and evidence synthesis}

Considerations

While synthesising available evidence to inform the consensus process, a number of important aspects require consideration: (1) Many of the included studies were performed in high volume hip surgeon practice settings. The patients in these studies have a high a priori probability of having a hip condition, leading to

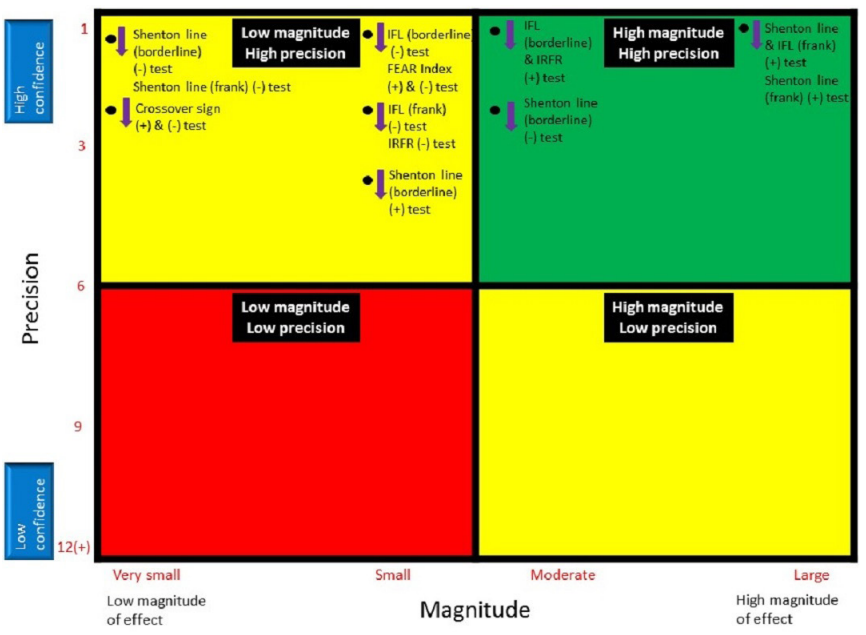

Figure 5 Magnitude and precision of imaging tests for diagnosis and screening of acetabular dysplasia and hip instability. (+), positive test; (-), negative test; FEAR, femoro-epiphyseal acetabular roof; IFL, iliofemoral line; IRFR, iliocapsularis-to-rectus femoris ratio.

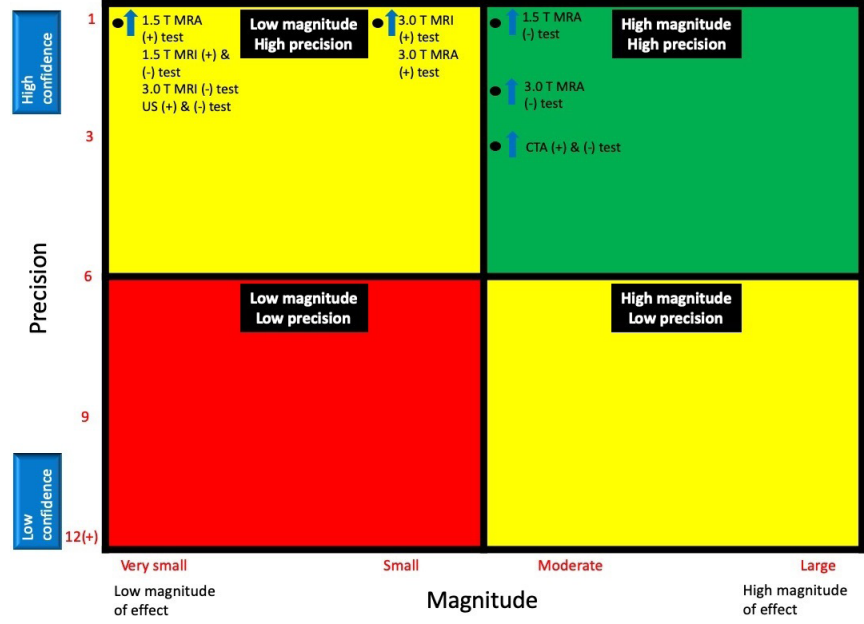

Figure 6 Magnitude and precision of imaging tests for diagnosis and screening of labral conditions. (+),positive test; $(-)$, negative test; CTA, CT arthrogram; MRA, magnetic resonance arthrogram; T, Tesla; US, ultrasound. Reference standards are listed in ().

a limited ability of an examination method or imaging modality to substantially alter the post-test probability. Future studies in general practice or physiotherapy settings might improve our understanding of the value of these tests. (2) Some conditions are defined by certain radiological appearances or cut-off values (eg, cam morphology where alpha angle is $>60$, pincer morphology where lateral centre edge angle (LCEA) is $>40$, acetabular dysplasia where LCEA is $<20$ ), but there is no consensus on specific imaging parameters to diagnose different hip conditions. As such, it is inappropriate to examine the value of imaging to assess the presence of a condition. (3) The third condition 'other' includes isolated or combined labral, chondral and ligamentum teres conditions as the most likely cause of hip-related nociception in the absence of distinct osseous morphology. This category should not be confused with the incidental imaging findings in asymptomatic people. Labral, chondral and/or ligamentum teres findings are common in asymptomatic individuals and can coexist in patients with FAI syndrome or acetabular dysplasia and/or hip instability.

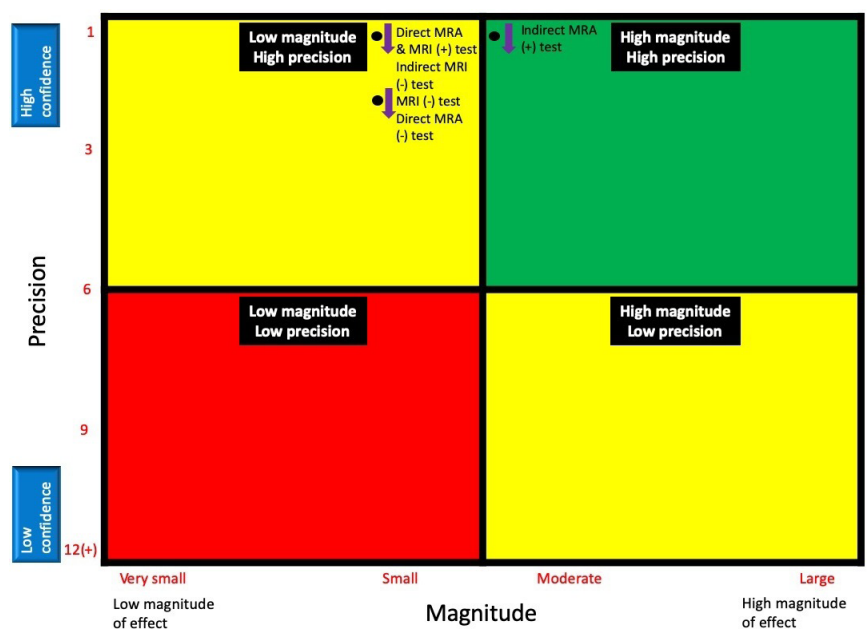

Figure 7 Magnitude and precision of imaging tests for diagnosis and screening of chondral conditions. (+),positive test; (-), negative test; MRA, magnetic resonance arthrogram. 


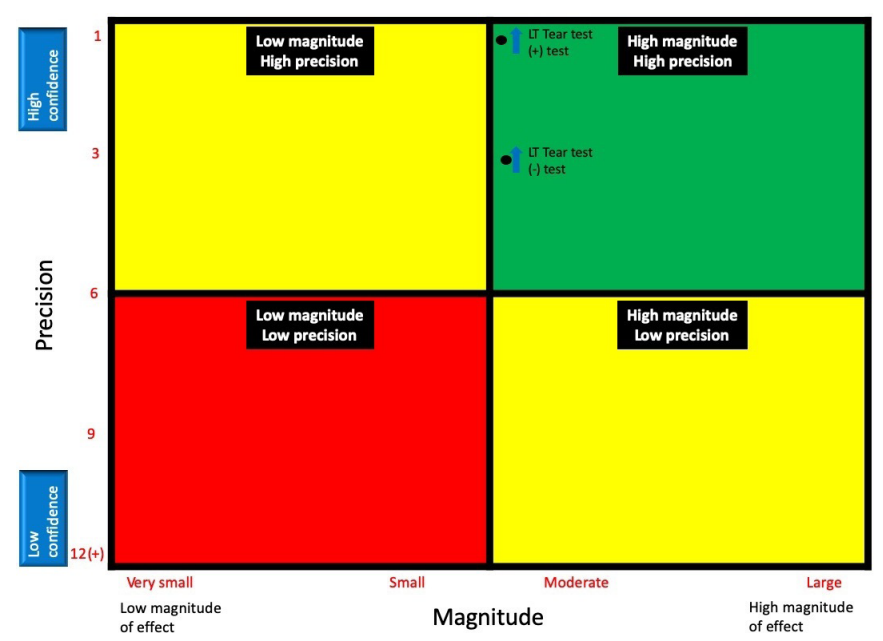

Figure 8 Magnitude and precision of clinical tests for diagnosis and screening of LT conditions. (+),positive test; (-), negative test; LT, ligamentum teres.

\section{Findings}

Both non-musculoskeletal and serious hip pathological conditions (eg, tumours, infections, stress fractures, SCFE), as well as competing musculoskeletal conditions (eg, lumbar spine) should be excluded when diagnosing hip-related pain in young and middle-aged active adults. Five hip conditions in young and middle-aged active adults presenting with hip-related pain met the inclusion criteria of this review: FAI syndrome; acetabular dysplasia and/or hip instability; labral, chondral and ligamentum teres conditions. We classified the last three conditions as 'other conditions'. It is important to note that labral, chondral and ligamentum teres pathology are common on imaging in asymptomatic people (and best-labelled 'incidental findings' in this context).

\section{Results of the consensus on topic 1 : classification of hip- related pain}

The IHiPRN delegates at the November 2018 Zurich consensus meeting discussed and voted on six recommendations: three recommendations for clinicians, one recommendation for clinicians and researchers and two for researchers. The final

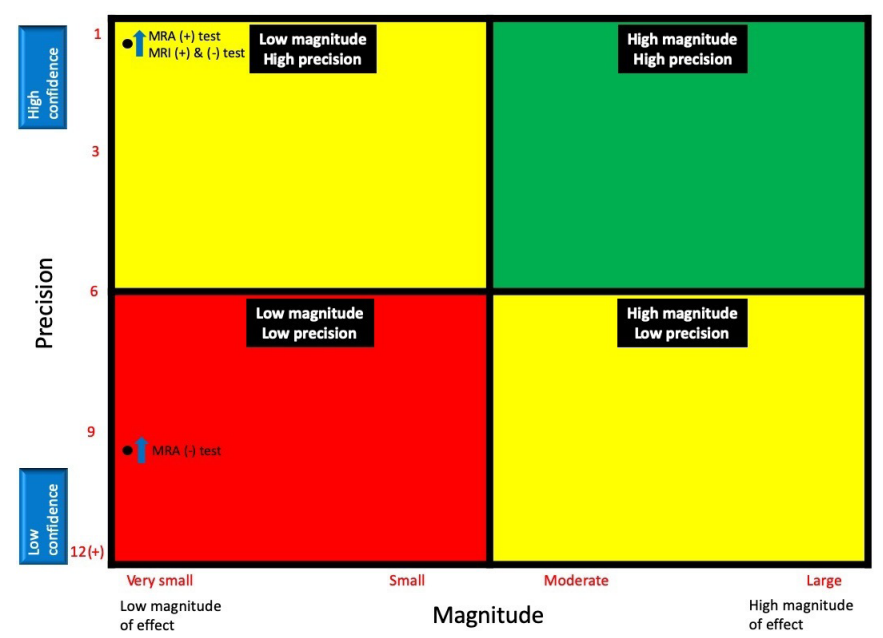

Figure 9 Magnitude and precision of imaging tests for diagnosis and screening of ligamentum teres conditions. (+), positive test; (-), negative test; MRA, magnetic resonance arthrogram. consensus recommendations and consensus voting results can be found in table 1 and figures 10-12. The consensus group considered all recommendations to be appropriate.

\section{DISCUSSION}

The IHiPRN consensus group met in Zurich in November 2018, and considered how to classify, define and diagnose hip disease in young and middle-aged active adults with hip-related pain as the main symptom. The working group conducted a scoping review, a useful framework to collate and summarise information on a broad topic, ${ }^{30}$ presented three clinical, one combined clinical and research and two research recommendations for consensus discussion and agreement.

This consensus paper builds on previous hip and groin pain consensus papers. ${ }^{2} 3$ It classifies hip disease in young and middle-aged active adults (with hip-related pain as the main symptom) into three hip conditions: (1) FAI syndrome, (2) acetabular dysplasia and/or hip instability and (3) other conditions without distinct osseous morphology. This final category includes patients with isolated or combined labral, chondral and ligamentum teres findings (including a range of distinct and overlapping pathologies including tears, cysts, erosions or hypertrophy). It may also include patients with no imaging findings. We also report the clinical utility of diagnostic findings for each of these conditions. It is important to acknowledge that these conditions often coexist. ${ }^{1138} 39$ We also acknowledge (but did not include here) other hip conditions with hip-related pain as the main symptom including osteoarthritis, infections, transient osteoporosis, stress fractures and tumours. We also acknowledge that currently diagnostic accuracy is limited to lower quality cohort studies with high pretest probability of disease. To what extent diagnostic accuracy is altered in studies of lower pretest probability is not understood, although one high-quality study with a lower pretest probability suggests the probability shift is similarly low. ${ }^{40}$ Other studies in settings of low pretest probability are necessary to determine the diagnostic accuracy of these various assessments in primary care setting.

Our evidence-informed classification of hip disease in young and middle-aged active adults classifies hip-related pain into three condition categories (once non-musculoskeletal and musculoskeletal serious hip pathology and competing body regions are excluded): FAI syndrome, acetabular dysplasia and/or hip instability, and other conditions, such as labral, chondral and/or ligamentum teres conditions. It is important, however, to appreciate that these conditions often coexist in patients with hip-related pain. ${ }^{39} 41$ These recommendations are not intended to be absolute for all young and middle-aged active adults with hip-related pain-a multifactorial condition. The clinical approach to hip disease in active adults with hip-related pain is highly contextual and should be integrated with clinical expertise using evidencebased practice and clinical decision-making models. ${ }^{42}$ Each of the consensus recommendations are discussed below and represented in the clinical case example (Box).

Clinical recommendation 1 (C1). A negative flexion adduction internal rotation (FADIR) test helps to rule out hip disease in young and middle-aged active adults presenting with hiprelated pain.

The diagnostic accuracy of the clinical assessment is mostly limited to clinical special tests and we cautiously recommended the FADIR test as the only clinical test for utilisation in attempt to help rule out potential hip-related pain. While the FADIR test is represented in our 'cautiously recommend' quadrant and the Thomas test is in the 'recommend' quadrant, the specifics of 
Table 1 Summary of final recommendations developed from evidence synthesis for voting at consensus meeting and scoring of consensus group

\begin{tabular}{|c|c|c|c|c|c|}
\hline \multicolumn{2}{|c|}{ Recommendations for clinicians } & \multirow{2}{*}{$\begin{array}{l}\text { Median } \\
9\end{array}$} & \multirow{2}{*}{$\frac{\text { IQR }}{8-9}$} & \multirow{2}{*}{ Mode } & \multirow{2}{*}{$\begin{array}{l}\text { Scoring } \\
\text { Appropriate }\end{array}$} \\
\hline $\mathrm{C} 1$ & $\begin{array}{l}\text { A negative FADIR test helps to rule out hip disease in young and middle-aged active adults presenting with } \\
\text { hip-related pain. }\end{array}$ & & & & \\
\hline $\mathrm{C} 2$ & $\begin{array}{l}\text { Diagnostic utility of imaging for hip disease in people with hip-related pain is limited; we suggest that } \\
\text { imaging always be combined with the patient's symptoms and clinical signs and should not be used in } \\
\text { isolation. }\end{array}$ & 9 & 9 & 9 & Appropriate \\
\hline $\mathrm{C} 3$ & $\begin{array}{l}\text { AP pelvis and lateral femoral head-neck radiographs are recommended to assist the diagnoses of } \\
\text { hip conditions associated with hip-related pain. MRI/MRA or CT scan are recommended where three- } \\
\text { dimensional morphological assessment is indicated or to evaluate intra-articular structures, such as the } \\
\text { labrum, cartilage and ligamentum teres. }\end{array}$ & 9 & 9 & 9 & Appropriate \\
\hline \multicolumn{2}{|c|}{ Recommendations for clinicians and researchers } & & & & Recommendation \\
\hline CR1 & $\begin{array}{l}\text { Hip-related pain may be further categorised after imaging into: } \\
\text { 1. FAl syndrome. } \\
\text { 2. Acetabular dysplasia and/or hip instability. } \\
\text { 3. Other conditions causing hip-related pain. This category includes soft-tissue conditions (labrum, } \\
\text { cartilage and ligamentum teres) without a specific bony morphology. }\end{array}$ & 9 & $8-9$ & 9 & Appropriate \\
\hline \multicolumn{2}{|c|}{ Recommendations for Researchers } & & & & Recommendation \\
\hline R1 & $\begin{array}{l}\text { Measures of bony morphology should be reported in detail. We recommend that bony morphology outcome } \\
\text { measures (such as the alpha angle or centre-edge angle) should be clearly defined, measured and reported } \\
\text { (eg, detailed methodological description, blinding, per hip/per person reporting with statistical correction as } \\
\text { appropriate, reliability measures) }\end{array}$ & 9 & 9 & 9 & Appropriate \\
\hline $\mathrm{R} 2$ & $\begin{array}{l}\text { Future research recommendations: We recommend large-scale, interdisciplinary research on aetiology and } \\
\text { prognosis for each of the listed hip-related pain conditions. (For example: (1) The relationship between bony } \\
\text { morphology and other factors related to these conditions or (2) Movement-related factors relative to each } \\
\text { hip-related pain condition.) }\end{array}$ & 9 & 9 & 9 & Appropriate \\
\hline
\end{tabular}

AP, anteroposterior; FADIR, flexion adduction internal rotation; FAl, femoroacetabular impingement; MRA, magnetic resonance arthrogram.

the studies examining these clinical tests is worthy of consideration. The Thomas test was examined in one high-quality study not specifically designed to determine diagnostic accuracy. The FADIR test has been examined in several studies; they are lowquality studies, most not designed to determine diagnostic accuracy. We therefore feel it is important to provide the clinician with the most appropriate clinical measure to potentially screen for all hip-related pain, based on the best current evidence, and recommend the FADIR test despite its noted limitations. Other clinical special tests (eg, Thomas test, prone instability test, ligamentum teres tear test) were considered by the expert group, and these tests have a:

- Very limited ability to confirm FAI syndrome by increasing post-test probability.

- Limited to substantial ability to help confirm acetabular dysplasia and/or hip instability in low-quality studies.

- Substantial ability to help rule in labral conditions in only one high-quality study.

- Unknown ability to rule in chondral conditions.

- Moderate post-test probability for confirming diagnosis of ligamentum teres conditions.

As the evidence for the clinical utility of these other tests is very limited or insufficient for ruling non-arthritic hip disease in or out in young and middle-aged active adults, we do not recommend their use by clinicians to diagnose the source of hip-related pain. In addition, we do not know the diagnostic accuracy of symptoms for conditions characterised by hip-related pain as mentioned earlier. The value of range-of-motion (ROM), muscle performance and other clinical tests for the diagnosis of a condition characterised by hip-related pain is unknown, as such tests currently lack diagnostic accuracy.

Clinical recommendation 2 (C2). Diagnostic utility of imaging for hip disease in young and middle-aged active adults presenting with hip-related pain is limited; we recommend that imaging should never be used in isolation but combined with the patient's symptoms and clinical signs (when making treatment and other relevant decisions).

In young and middle-aged active adults with hip disease, this recommendation on diagnostic utility of imaging should underpin any agreement on diagnostic criteria. Diagnostic imaging demonstrates post-test probability shifts ranging from very small to substantial for the diagnosis of FAI syndrome, and acetabular dysplasia and/or hip instability, and small to moderate shifts at best for labral, chondral and ligamentum teres pathology. Diagnostic imaging has a very limited ability to confirm the diagnosis of a particular condition as the cause of pain. As such, we agreed with previous recommendations that the diagnostic criteria should include appropriate diagnostic imaging, but also consideration of symptoms and clinical signs. ${ }^{32}$ Diagnosis of the primary cause of hip-related pain should never be made using imaging alone. The next step in the research process is to evaluate the performance of these diagnostic criteria, which primarily includes clinical signs and diagnostic imaging for the various hip conditions with hip-related pain as the primary symptom.

Clinical recommendation 3 (C3). We recommend anteroposterior (AP) pelvis and lateral femoral head-neck radiographs to assist in diagnosing the hip conditions characterised by hiprelated pain, and MRI/magnetic resonance arthrogram (MRA) or CT scan where three-dimensional morphological assessment is indicated.

Our recommendation is consistent with prior recommendation $^{3}$ that the initial diagnostic imaging should at least include AP pelvic and lateral femoral head-neck view radiographs. The latter can, for example, include a Dunn view, a frog-leg (also known as Lauenstein) view or a cross-table view. This will provide the clinician with an understanding of the underlying morphology of the hip, which may be relevant for the patient presenting with hip-related pain. When indicated (clinically or in research), advanced imaging can be used for more clarity on 
three-dimensional morphology or to assess intra-articular structures. For intra-articular structures such as the labrum, cartilage and ligamentum teres, we recommend MRI/MRA or CT arthrogram scan. Incidental intra-articular findings are common in asymptomatic individuals and should be managed with the appropriate clinical sensitivity. ${ }^{14344}$ Again, caution is suggested as the clinical utility of diagnostic imaging can have only small shifts in post-test probability as a result of either positive or negative findings. ${ }^{45-47}$ As such, a comprehensive clinical examination, including screening for other conditions that might present with hip-related pain (eg, of the spine and pelvis), is required, and the diagnosis of the cause of hip-related pain should never be made on imaging alone. 2223

Clinical and research recommendation 1 (CR1). Hip-related pain may be further categorised after imaging into: (1) FAI syndrome; (2) acetabular dysplasia and/or hip instability and (3) other conditions causing hip-related pain. This last category could include soft-tissue conditions without specific bony morphology.

For both clinical and research purposes, imaging can help to categorise hip disease in young and middle-aged active adults presenting with hip-related pain into the following conditions: (1) FAI syndrome, (2) acetabular dysplasia and/or hip instability and (3) other conditions causing hip-related pain without specific bony morphology, such as labral, chondral and/or ligamentum teres conditions. Many hip-related pain conditions-especially labral and chondral conditions-coexist with FAI syndrome and acetabular dysplasia and/or hip instability. ${ }^{39} 41$ The third category (similar to previous recommendations) ${ }^{2}$ is less distinct with variable clinical presentations and includes intra-articular conditions without a distinct bony morphology. We acknowledge that special tests ${ }^{48}$ and imaging ${ }^{43}{ }^{44}$ have high rates of falsepositive findings and that patients can present with symptoms and signs similar to that of FAI syndrome, but have normal bony morphology. ${ }^{49}$ The third category of 'other conditions' will include these patients. Finally, we acknowledge that patients may present with pain, but normal morphology or no findings consistent with other pathology on imaging can explain nociception. Standardised and well-reported methods in research, such as those suggested in this recommendation, will reduce research heterogeneity, while improving the accuracy of summary estimates in meta-analyses. ${ }^{39}$

Research recommendation 1. Measures of bony morphology should be reported in detail. We recommend that bony morphology outcome measures (such as the alpha angle or centre-edge angle) should be clearly defined, measured and reported (eg, detailed methodological description; blinding; per hip/per person reporting with statistical correction as appropriate; reliability measures).

We recommend that researchers provide the following as part of the methodology section of research reports when using bony morphology imaging outcome measures: (1) define the outcome measure; (2) report a reliable and complete description of imaging methodology and details on how the outcome is measured and (3) provide detail of the statistical analysis used. ${ }^{50}$

Research recommendation 2. Future research recommendations: We recommend large-scale, interdisciplinary research on aetiology and prognosis for each of the listed three hip conditions (characterised by hip-related pain).

Studies that examine the aetiology and prognosis of the three hip conditions are critical if we are to effectively classify, define and diagnose hip-related pain in young and middle-aged active adults. To classify and define each condition, we must identify the aetiology of the condition. Understanding the aetiology of
A hypothetical clinical case example (not requiring patient consent statement) describing the implementation of recommendations $\mathrm{C} 1$, C2, C3 and CR1 into clinical practice.

Patient described is a 25-year-old male football player with a 6-month history of anterior hip and groin pain, aggravated by kicking and changing direction activities.

C1: A negative flexion-adduction-internal rotation (FADIR) test may help to rule out hip in young and middle-aged active adults presenting with hip-related pain. The FADIR test should be performed as part of a comprehensive physical examination. If the test is negative, the clinician could consider that the source of pain is possibly not the hip joint. If the test is positive, the hip may be a source of nociception, and further assessment is required.

The patient has a positive FADIR test.

C2: Diagnostic utility of imaging for hip disease in young and middle-aged active adults presenting with hip-related pain is limited; it should be interpreted together with the patient's symptoms and clinical signs. His anteroposterior (AP) pelvis and lateral radiographs have been obtained. The report states the presence of cam morphology. While these findings may be contributing to his pain and match the clinical presentation, the clinician should be aware of the prevalence of cam morphology $(66 \%)$ in athletes. ${ }^{1}$

C3: We recommend AP pelvis and lateral femoral headneck radiographs to assist in diagnosing the hip conditions characterised by hip-related pain, and MRI or CT scan where three-dimensional morphological assessment is indicated. He has a positive FADIR test and has cam morphology on radiographs. In consultation with his medical team, he has decided to pursue surgical options to address his continued hip pain. Referral for MRI or CT scan may assist in identifying labral, chondral or other hip conditions when clinically indicated; especially if nonsurgical treatment such as physiotherapy has not succeeded and surgery is being considered, MRI may be necessary to better plan surgery.

CR1: Hip-related pain may be further categorised after imaging into: (1) femoroacetabularimpingement (FAI) syndrome, (2) acetabular dysplasia and/or hip instability and (3) other conditions causing hip-related pain. This last category could include soft-tissue conditions without specific bony morphology. The comprehensive subjective assessment of the patient's complaints/pain, physical examination and imaging undertaken suggest that he may have FAI syndrome (positive FADIR test, cam morphology and labral lesions on MRI scan). Categorising his condition as FAl syndrome may assist him in understanding his condition and may aid the shared decision-making process regarding appropriate management decisions.

the conditions will eventually enable effective primary, secondary and tertiary prevention strategies to be developed. We must conduct studies that examine the prognosis of young and middleaged people with hip-related pain. We can determine prognosis by conducting research that explores the relationship of each of the three conditions with other factors, such as pain, other symptoms, psychological factors, socioeconomic factors and physical impairments. We will then be able to begin to develop accurate assessment processes and effective, targeted treatments. The development of studies of aetiology and prognosis can be organised into a logical, step-by-step process including ${ }^{51-53}$. 1. Aetiology 
A. Agreeing on the classification of hip disease presenting with hip-related pain (ie, the different conditions).

B. Agreeing on the diagnostic criteria for each hip condition.

2. Prognosis

A. Determining the performance of these criteria (eg, diagnostic accuracy of clinical and radiological examination).

B. Investigating the impact of the diagnostic components of a specific hip condition on diagnostic or prognostic thinking (eg, stratification of patients into high and low risk; determining the prognostic value of the different conditions on long-term outcomes, for example, osteoarthritis).

C. Determining the impact on diagnostic or therapeutic strategies (eg, determining if advanced imaging is required for diagnosis, agreeing on an appropriate treatment strategy).

3. Financial, health-related, and patient and provider satisfaction outcomes (eg, cost-effectiveness studies, implementation of satisfaction outcome scores in the clinic and report in treatment outcome studies).

Research investigating the hip conditions in young and middleaged active adults with hip-related pain has not completed this logical process. Additionally, while we point out that progress has been made with steps 1 and 3, the remaining steps are largely ignored ${ }^{54}$ with current literature focusing on outcomes for treatment strategies. ${ }^{55} 56$ Thus, we recommend large-scale, prospective and interdisciplinary research on aetiology and prognosis for each of the three primary condition categories of hip disease with hip-related pain as the main symptom and their associated bony morphologies and soft-tissue pathology.

\section{Limitations}

Several limitations require consideration regarding the clinical and research recommendations discussed in this paper. The group of participants who voted on these recommendations at the first IHiPRN meeting in Zurich included many different professions, and diversity in research and clinical experience. However, due to the invitation process and budgetary and logistical limitations, the group cannot be considered fully inclusive. Therefore, the contributions were limited to the participants who were part of the consensus process. We acknowledge the possibility of bias, and homogeneity of expert opinion, and that future consensus meetings will aim to include a broader range of experts, as well as consumers (patient representatives).

The extremely high level of agreement demonstrated in figures 10-12 may represent a ceiling effect or bias. In previous work using similar RAND Appropriateness Method scales, ${ }^{57}$ that bias can be reduced by having an 'effective group leadership'. The design of IHiPRN, working groups, collaborations

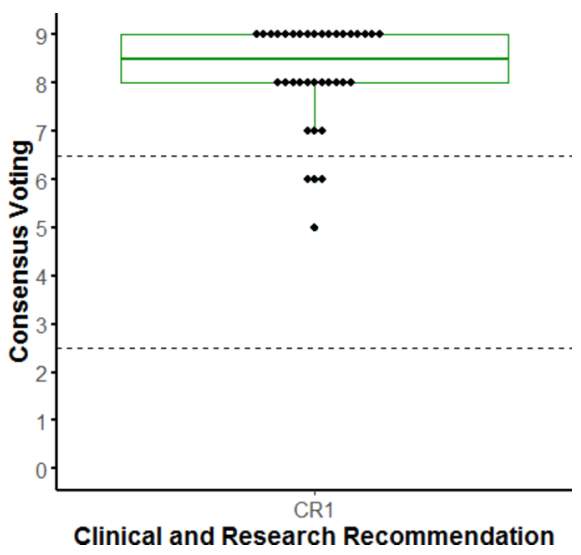

Figure 11 Summary of consensus voting on clinical/research recommendations.

premeeting, during and postmeeting all were focused on limiting any potential bias.

This consensus is based on a scoping review of only English language studies with no quality assessment of included studies. It is possible that we missed important studies in other languages and included low-quality studies or reviews. Interpretation of study quality was inferred from the included systematic reviews.

Consensus statement voting did not cover all aspects of this paper. For example, studies without quality assessment (eg, studies on condition definition and epidemiology) were not addressed in consensus statements.

We acknowledge that other hip conditions not addressed in this paper can also lead to hip-related pain in young active adults. The conditions described are most prevalent in young and middle-aged active adults with hip-related pain.

Finally, there are a number of concerns with diagnostic accuracy studies, including: (1) typically performed in settings of high pretest probability (eg, surgical populations), (2) the majority of these studies are low-quality cohort studies with a high risk of bias in many quality domains-diagnostic accuracy might therefore be overestimated ${ }^{58} 59$ and (3) several patients with hip-related pain might have more than one hip condition (on imaging or in surgery). ${ }^{11} 38{ }^{39} \mathrm{It}$ is worth mentioning that caution is warranted in interpretation of these consensus statements knowing that the information available is based on biased high-prevalence, lower-level cohort evidence.

\section{CONCLUSION}

The first IHiPRN Consensus Meeting was held in Zurich, Switzerland, in November 2018. The IHiPRN participants developed and gained consensus on three clinical recommendations, one

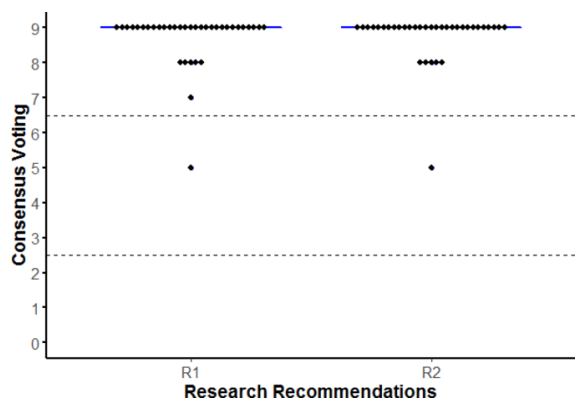

Figure 12 Summary of consensus voting on research recommendations.

Figure 10 Summary of consensus voting on clinical recommendations. 
clinical and research recommendation and two research recommendations, all of which were an amalgamation of best available evidence and expert opinion. Both non-musculoskeletal and serious hip pathological conditions (eg, tumours, infections, SCFE), as well as competing musculoskeletal conditions (eg, lumbar spine) should first be excluded before categorising hip disease in young and middle-aged active adults presenting with hip-related pain. Once these are excluded, hip-related pain should be categorised into: (1) FAI syndrome, (2) acetabular dysplasia and/or hip instability and (3) other conditions without bony morphology causing hip-related pain, such as chondral, labral and ligamentum teres conditions. The diagnostic utility of clinical and diagnostic imaging examination in isolation is limited; therefore, we recommend a comprehensive examination of patient symptoms, clinical signs and diagnostic imaging. We recognise that the diagnostic capability of matching symptoms, clinical signs and diagnostic imaging is unknown for patients with hip-related pain. Diagnostic imaging for hip-related pain should at least include AP pelvis and a lateral femoral head-neck view radiographs; advanced imaging is recommended when further assessment of intra-articular structures or morphology is indicated. Clearly this is an area requiring further research examination to provide both the clinician and researcher greater clarity regarding imaging utility.

Research studies reporting bony morphology should provide clear definitions and detailed imaging and reporting methods. We recommend large-scale, prospective and interdisciplinary research on aetiology and prognosis of hip-related pain where the overlapping nature of the various conditions is considered.

\section{Author affiliations}

${ }^{1}$ Orthopedic Surgery, Duke University Medical Center, Durham, North Carolina, USA ${ }^{2}$ Department of Orthopaedic Surgery, Erasmus, MC, University Medical Center, Rotterdam, Netherlands

${ }^{3}$ La Trobe Sport and Exercise Medicine Research Centre, La Trobe University, Melbourne, Victoria, Australia

${ }^{4}$ Orthopaedics, Erasmus MC Center for Groin Injuries, Rotterdam, The Netherlands

${ }^{5}$ Sports Groin Pain Centre, Aspetar Hospital, Doha, Qatar

${ }^{6}$ Elite Sports Imaging SL, Madrid, Spain

${ }^{7}$ Musculoskeletal Radiology, Corades, LLC, Brookline, MA, United States

${ }^{8}$ Sport Sciences, Deparment of Health Sciences, Lund University, Lund, Sweden

${ }^{9}$ Sports Orthopaedic Research Center-Copenhagen (SORC-C), Arthroscopic Center, Department of Orthopedic Surgery, Copenhagen University Hospital, AmagerHvidovre, Denmark

${ }^{10}$ Oslo University Hospital, Oslo, Norway

${ }^{11}$ Orthopaedics - Medical School, University of Warwick, Coventry, UK

${ }^{12}$ Warwick Medical School, University of Warwick, Coventry, UK

${ }^{13} \mathrm{Centre}$ for Hip Health and Mobility, The University of British Columbia, Vancouver,

British Columbia, Canada

${ }^{14}$ Research, Schulthess Clinic Human Performance Lab, Zurich, Switzerland

${ }^{15}$ Physical Therapy, Washington University, St. Louis, Missouri, USA

${ }^{16}$ Human Performance Lab, Schulthess Clinic, Zurich, Switzerland

${ }^{17}$ Laboratory of Exercise and Health, ETH Zurich, Schwerzenbach, Switzerland

${ }^{18} \mathrm{Griffith}$ Centre of Biomedical and Rehabilitation Engineering (GCORE), Menzies

Health Institute Queensland Griffith University, Schoold of Allied Health Sciences,

Griffith, Queensland, Australia

${ }^{19}$ School of Health and Rehabilitation Sciences, Ohio State University Wexner Medical

Center, Columbus, Ohio, USA

${ }^{20}$ Athlete Availability, Australian Institute of Sport, Canberra, Australian Capital

Territory, Australia

${ }^{21}$ Research into Sport and Exercise, University of Canberra, Canberra, Bruce,

Australian Capital Territory, Australia

${ }^{22}$ Alfred Health, Melbourne, Victoria, Australia

${ }^{23}$ School of Health and Rehabilitation Sciences, University of Queensland, Brisbane, Queensland, Australia

${ }^{24}$ Swiss Olympic Medical Center, Hopital de la Tour, Meyrin, Geneva, Switzerland

${ }^{25}$ Sports Medicine, Centre Hospitalier Universitaire Vaudois, Lausanne, Switzerland

${ }^{26}$ Department of Orthopaedic Surgery, University of Oxford, Institute of

Musculoskeletal Sciences, Oxford, United Kingdom

${ }^{27}$ Physical Therapy, University of British Columbia, Vancouver, British Columbia, Canada
${ }^{28}$ Faculty of Health, University of Technology Sydney, Sydney, New South Wales, Australia

${ }^{29}$ School of Health and Rehabilitation Sciences, University of Queensland, Brisbane, Queensland, Australia

${ }^{30}$ Department of Orthopaedics, Schulthess Klinik, Zurich, Switzerland

${ }^{31}$ Physical Therapy \& Athletic Training, Boston University, Boston, Massachusetts, USA

${ }^{32}$ Physiotherapy, HES-SO Valais, University of Applied Sciences Western Switzerland, Loeche-les-Bains, Valais, Switzerland

${ }^{33}$ Oslo Sports Trauma Research Center, Oslo, Norway

${ }^{34}$ Division of Orthopedic Surgery, Oslo University Hospital, Oslo, Norway

${ }^{35}$ Department of Sports Medicine, Norwegian School of Sport Sciences, Oslo, Norway

${ }^{36}$ Aspetar Qatar Orthopaedic and Sports Medicine Hospital, Doha, Qatar

${ }^{37}$ Department of Health Sciences, Lund University, Lund, Sweden

${ }^{38}$ Weill Cornell Medicine, Doha, Qatar

Twitter Michael P Reiman @MikeReiman, Rintje Agricola @RintjeAgricola, Joanne L Kemp @JoanneLKemp, Joshua J Heerey @JHeerey, Pim van Klij @pimvklij, Ara Kassarjian @akassarjian, Andrea Britt Mosler @AndreaBMosler, Eva Ageberg @EvaAgeberg, Damian Griffin @DamianGriffin, Nicola C Casartelli @NicCasartelli, Laura E Diamond @lauradiamond05, Stephanie Di Stasi @S_DiStasi, Michael Drew @ mickdrew, Daniel J Friedman @ddfriedman, Boris Gojanovic @drsportsante, Marcie Harris-Hayes @MHarrisHayes, Franco M Impellizzeri @francoimpell, Lasse Ishøi @Lasselshoei, Matthew G King @mattgmking1, Peter R Lawrenson @PeteLawrenson, Cara L Lewis @ProfCaraLewis, Håvard Moksnes@HMoksnes, Mark James Scholes@MarkScholes85, Adam I Semciw @ASemciw, Andreas Serner @aserner, Kristian Thorborg @KThorborg, Tobias Wörner @Wuninho and Hendrik Paulus Dijkstra @DrPaulDijkstra

Contributors The concept was developed by JLK, ABM, KMC, KMK, CLL and MB. All authors contributed to the material presented and approved the final version of the manuscript. All authors contributed to the consensus voting and approval of manuscript.

Funding The authors have not declared a specific grant for this research from any funding agency in the public, commercial or not-for-profit sectors.

Competing interests None declared.

Patient consent for publication Not required.

Provenance and peer review Not commissioned; externally peer reviewed.

Data availability statement All data relevant to the study are included in the article or uploaded as supplementary information

\section{ORCID iDs}

Michael P Reiman http://orcid.org/0000-0003-4557-3446

Joanne L Kemp http://orcid.org/0000-0002-9234-1923

Joshua J Heerey http://orcid.org/0000-0001-8691-1830

Pim van Klij http://orcid.org/0000-0002-6515-8322

Andrea Britt Mosler http://orcid.org/0000-0001-7353-2583

Eva Ageberg http://orcid.org/0000-0002-8639-3006

Kristian Marstrand Warholm http://orcid.org/0000-0003-0978-0667

Karim M Khan http://orcid.org/0000-0002-9976-0258

Kay M Crossley http://orcid.org/0000-0001-5892-129X

Nicola C Casartelli http://orcid.org/0000-0002-1280-866X

Laura E Diamond http://orcid.org/0000-0002-2197-1856

Stephanie Di Stasi http://orcid.org/0000-0001-6302-4147

Boris Gojanovic http://orcid.org/0000-0001-5075-9371

Marcie Harris-Hayes http://orcid.org/0000-0003-4274-1651

Franco M Impellizzeri http://orcid.org/0000-0002-1703-2573

Lasse Ishøi http://orcid.org/0000-0002-2716-6567

Matthew G King http://orcid.org/0000-0003-0470-5924

Peter R Lawrenson http://orcid.org/0000-0002-6479-6840

Cara L Lewis http://orcid.org/0000-0002-9888-4902

Mark James Scholes http://orcid.org/0000-0001-9216-1597

Andreas Serner http://orcid.org/0000-0003-4308-901X

Kristian Thorborg http://orcid.org/0000-0001-9102-4515

Tobias Wörner http://orcid.org/0000-0001-5555-0876

\section{REFERENCES}

1 Mascarenhas VV, Rego P, Dantas P, et al. Imaging prevalence of femoroacetabular impingement in symptomatic patients, athletes, and asymptomatic individuals: a systematic review. Eur J Radiol 2016;85:73-95.

2 Weir A, Brukner P, Delahunt E, et al. Doha agreement meeting on terminology and definitions in groin pain in athletes. Br J Sports Med 2015;49:768-74.

3 Griffin DR, Dickenson EJ, O'Donnell J, et al. The Warwick agreement on femoroacetabular impingement syndrome (FAI syndrome): an international consensus statement. Br J Sports Med 2016;50:1169-76. 
4 Nepple JJ, Prather H, Trousdale RT, et al. Clinical diagnosis of femoroacetabular impingement. J Am Acad Orthop Surg 2013;21:S16-19.

5 Nepple JJ, Prather H, Trousdale RT, et al. Diagnostic imaging of femoroacetabular impingement. J Am Acad Orthop Surg 2013;21:S20-6.

6 Sankar WN, Nevitt M, Parvizi J, et al. Femoroacetabular impingement: defining the condition and its role in the pathophysiology of osteoarthritis. J Am Acad Orthop Surg 2013;21:S7-15

7 Reiman MP, Thorborg K, Covington K, et al. Important clinical descriptors to include in the examination and assessment of patients with femoroacetabular impingement syndrome: an international and multi-disciplinary Delphi survey. Knee Surg Sports Traumatol Arthrosc 2017:25:1975-86.

8 Reiman MP, Goode AP, Cook CE, et al. Diagnostic accuracy of clinical tests for the diagnosis of hip femoroacetabular impingement/labral tear: a systematic review with meta-analysis. Br J Sports Med 2015;49:811.

9 Tijssen M, van Cingel R, Willemsen L, et al. Diagnostics of femoroacetabular impingement and Labral pathology of the hip: a systematic review of the accuracy and validity of physical tests. Arthroscopy 2012:28:860-71.

10 Wilkin GP, Ibrahim MM, Smit KM, et al. A contemporary definition of hip dysplasia and structural instability: toward a comprehensive classification for acetabular dysplasia. J Arthroplasty 2017;32:520-7.

11 O'Donnell J, Economopoulos K, Singh P, et al. The ligamentum teres test: a nove and effective test in diagnosing tears of the ligamentum teres. Am J Sports Med 2014;42:138-43.

12 Agricola R, Heijboer MP, Bierma-Zeinstra SMA, et al. Cam impingement causes osteoarthritis of the hip: a nationwide prospective cohort study (check). Ann Rheum Dis 2013:72:918-23.

13 Saberi Hosnijeh F, Zuiderwijk ME, Versteeg M, et al. Cam deformity and acetabular dysplasia as risk factors for hip osteoarthritis. Arthritis Rheumatol 2017:69:86-93.

14 Clohisy JC, Baca G, Beaule PE, et al. Descriptive epidemiology of femoroacetabular impingement: a North American cohort of patients undergoing surgery. Am J Sports Med 2013:41:1348-56.

15 Byrd JW. Evaluation of the hip: history and physical examination. N Am J Sports Phys Ther 2007:2:231-40

16 Kivlan BR, Nho SJ, Christoforetti JJ, et al. Multicenter outcomes after hip arthroscopy: epidemiology (mash Study Group). what are we seeing in the office, and who are we choosing to treat? Am J Orthop 2017;46:35-41.

17 McCarthy JC, Busconi B. The role of hip arthroscopy in the diagnosis and treatment of hip disease. Orthopedics 1995;18:753-6.

18 Narvani AA, Tsiridis E, Kendall S, et al. A preliminary report on prevalence of acetabular labrum tears in sports patients with groin pain. Knee Surg Sports Traumatol Arthrosc 2003:11:403-8.

19 Keeney JA, Peelle MW, Jackson J, et al. Magnetic resonance arthrography versus arthroscopy in the evaluation of articular hip pathology. Clin Orthop Relat Res 2004:429:163-9.

20 Genda E, Iwasaki N, Li G, et al. Normal hip joint contact pressure distribution in single-leg standing —effect of gender and anatomic parameters.J Biomech 2001;34:895-905

21 Henak CR, Abraham CL, Anderson AE, et al. Patient-Specific analysis of cartilage and labrum mechanics in human hips with acetabular dysplasia. Osteoarthritis Cartilage 2014:22:210-7.

22 Thorborg K, Reiman MP, Weir A, et al. Clinical examination, diagnostic imaging, and testing of athletes with groin pain: an evidence-based approach to effective management. J Orthop Sports Phys Ther 2018:48:239-49.

23 Reiman MP, Thorborg K. Clinical examination and physical assessment of hip jointrelated pain in athletes. Int J Sports Phys Ther 2014:9:737-55.

24 Orchard J, Seward H, Orchard J. Afl injury report, 2012. Available: http://www. afl.com. au/staticfile/AFL\%20Tenant/AFL/Files/AFLInjuryReportFor2012.pdf [Accessed 529 14].

25 Orchard JW, Seward H, Orchard JJ. Results of 2 decades of injury surveillance and public release of data in the Australian football League. Am I Sports Med 2013:41:734-41.

26 Reiman MP, Thorborg K. Femoroacetabular impingement surgery: are we moving too fast and too far beyond the evidence? Br J Sports Med 2015;49:782-4

27 Reiman MP, Thorborg K, Hölmich P. Femoroacetabular impingement surgery is on the Rise—But what is the next step? J Orthop Sports Phys Ther 2016;46:406-8.

28 Cvetanovich GL, Chalmers PN, Levy DM, et al. Hip arthroscopy surgical volume trends and 30-day postoperative complications. Arthroscopy 2016;32:1286-92.

29 Arksey H, O'Malley L. Scoping studies: towards a methodological framework. Int I Soc Res Methodol 2005;8:19-32.

30 Murray A, Daines L, Archibald D, et al. The relationship and effects of golf on physical and mental health: a scoping review protocol. Br I Sports Med 2016;50:647-50.

31 Collins NJ, Barton CJ, van Middelkoop M, et al. 2018 consensus statement on exercise therapy and physical interventions (orthoses, taping and manual therapy) to treat patellofemoral pain: recommendations from the 5 th International Patellofemoral pain research retreat, gold Coast, Australia, 2017. Br J Sports Med 2018;52:1170-8.

32 Crossley KM, van Middelkoop M, Callaghan MJ, et al. 2016 Patellofemoral pain consensus statement from the 4 th International Patellofemoral pain research retreat, Manchester. Part 2: recommended physical interventions (exercise, taping, bracing, foot orthoses and combined interventions). Br J Sports Med 2016;50:844-52

33 Brouwers MC, Kerkvliet K, Spithoff K, et al. The agree reporting checklist: a tool to improve reporting of clinical practice guidelines. BMJ 2016;352:11152.

34 Jaeschke R, Guyatt GH, Sackett DL. Users' guides to the medical literature. III. How to use an article about a diagnostic test. B. what are the results and will they help me in caring for my patients? the evidence-based medicine Working group. JAMA 1994;271:703-7.

35 Poole C. Low P-values or narrow confidence intervals: which are more durable? Epidemiology 2001;12:291-4.

36 Whiting P, Rutjes AWS, Reitsma JB, et al. The development of QUADAS: a tool for the quality assessment of studies of diagnostic accuracy included in systematic reviews. BMC Med Res Methodol 2003;3:25.

37 Whiting PFet al. QUADAS-2: a revised tool for the quality assessment of diagnostic accuracy studies. Ann Intern Med 2011;155:529-36.

38 Kemp JL, Makdissi M, Schache AG, et al. Hip chondropathy at arthroscopy: prevalence and relationship to labral pathology, femoroacetabular impingement and patientreported outcomes. Br I Sports Med 2014;48:1102-7.

39 Reiman MP, Peters S, Sylvain J, et al. Prevalence and consistency in surgical outcome reporting for femoroacetabular impingement syndrome: a scoping review. Arthroscopy 2018:34:1319-28.

40 Ayeni $\mathrm{O}$, Chu R, Hetaimish B, et al. A painful squat test provides limited diagnostic utility in CAM-type femoroacetabular impingement. Knee Surg Sports Traumatol Arthrosc 2014;22:806-11

41 Peters S, Laing A, Emerson C, et al. Surgical criteria for femoroacetabular impingement syndrome: a scoping review. Br J Sports Med 2017;51:1605-10.

42 Elwyn G, Edwards A, Kinnersley $P$, et al. Shared decision making and the concept of equipoise: the competences of involving patients in healthcare choices. Br J Gen Pract 2000:50:892-9.

43 Heerey JJ, Kemp JL, Mosler AB, et al. What is the prevalence of imaging-defined intraarticular hip pathologies in people with and without pain? A systematic review and meta-analysis. Br J Sports Med 2018;52:581-93.

44 Frank JM, Harris JD, Erickson BJ, et al. Prevalence of femoroacetabular impingement imaging findings in asymptomatic volunteers: a systematic review. Arthroscopy 2015;31:1199-204.

45 Reiman MP, Thorborg K, Goode AP, et al. Diagnostic accuracy of imaging modalities and injection techniques for the diagnosis of femoroacetabular Impingement/Labral tear: a systematic review with meta-analysis. Am I Sports Med 2017:45:2665-77.

46 Saied AM, Redant C, El-Batouty M, et al. Accuracy of magnetic resonance studies in the detection of chondral and labral lesions in femoroacetabular impingement: systematic review and meta-analysis. BMC Musculoskelet Disord 2017;18:83.

47 Shakoor D, Farahani SJ, Hafezi-Nejad N, et al. Lesions of ligamentum teres: diagnostic performance of MRI and Mr Arthrography-A systematic review and meta-analysis. AJR Am J Roentgenol 2018:W1-12 (published Online First: 2018/05/25).

48 Casartelli NC, Brunner R, Maffiuletti NA, et al. The FADIR test accuracy for screening CAM and pincer morphology in youth ice hockey players. Journal of Science and Medicine in Sport 2018;21:134-8.

49 Mardones R, Giai Via A, Tomic A, et al. Arthroscopic release of iliopsoas tendon in patients with femoro-acetabular impingement: clinical results at mid-term follow-up. Muscle Ligaments and Tendons J 2016:06:378-83.

50 Hayden JA, van der Windt DA, Cartwright JL, et al. Assessing bias in studies of prognostic factors. Ann Intern Med 2013;158:280-6.

51 Jutel A. Sociology of diagnosis: a preliminary review. Sociol Health IIIn 2009;31:278-99.

52 Douglas PS. Improving imaging: our professional imperative. J Am Coll Cardiol 2006:48:2152-5

53 Newman-Toker DE. A unified conceptual model for diagnostic errors: underdiagnosis, overdiagnosis, and misdiagnosis. Diagnosis 2014;1:43-8.

54 Cunningham DJ, Paranjape CS, Harris JD, et al. Advanced imaging adds little value in the diagnosis of femoroacetabular impingement syndrome. J Bone Joint Surg Am 2017:99:e133

55 Lodhia P, Gui C, Chandrasekaran S, et al. The economic impact of acetabular Labral tears: a cost-effectiveness analysis comparing hip arthroscopic surgery and structured rehabilitation alone in patients without osteoarthritis. Am J Sports Med 2016;44:1771-80

56 Mather RC, Nho SJ, Federer A, et al. Effects of arthroscopy for femoroacetabular impingement syndrome on quality of life and economic outcomes. Am I Sports Med 2018:46:1205-13.

57 McAlindon TE, Bannuru RR, Sullivan MC, et al. OARSI guidelines for the non-surgical management of knee osteoarthritis. Osteoarthritis and Cartilage 2014:22:363-88.

58 Whiting PF, Rutjes AWS, Westwood ME, et al. A systematic review classifies sources of bias and variation in diagnostic test accuracy studies. J Clin Epidemiol 2013:66:1093-104.

59 Rutjes AWSet al. Evidence of bias and variation in diagnostic accuracy studies. Can Med Assoc J 2006:174:469-76. 